\title{
Einsatzmöglichkeiten von Geoinformationssystemen in der Siedlungswasserwirtschaft am Beispiel Einbindung dezentraler Entwässerungssysteme zur Entlastung des städtischen Abwassernetzes
}

\author{
Yannick Back · Jonatan Zischg · Magnus Bremer · Martin Rutzinger · Manfred Kleidorfer \\ Online publiziert: 6. Mai 2019 \\ (c) Der/die Autor(en) 2019
}

Zusammenfassung Im Zuge der voranschreitenden Digitalisierung und der damit verbundenen steigenden Verfügbarkeit von digitalen Daten gewinnen geografische Informationssysteme zur Analyse und Verwaltung von Geodaten in vielen technisch-wissenschaftlichen Bereichen, einschließlich der Siedlungswasserwirtschaft, immer mehr an Bedeutung. Rund $90 \%$ der weltweit verfügbaren digitalen Daten wurden allein in den letzten zwei Jahren generiert. Die steigende Verfügbarkeit von digitalen Daten bietet zum einen ein neues Potenzial von Anwendungsmöglichkeiten, andererseits aber für den/die Endbenutzer/in und Planer/in Unklarheiten über den Informationsgehalt. Ziel dieser Arbeit ist es, einen Überblick über geore-

\section{Abb. 1 und Tab. 1 wurden}

wiederverwendet/übersetzt aus: Toth und Józków (2016): Remote sensing platforms and sensors: A survey. ISPRS Journal of Photogrammetry and Remote Sensing (115): 22-36 (mit Erlaubnis von Elsevier).

Y. Back, MSc ( $₫) \cdot$ DI J. Zischg • Assoz.-Prof. DI Dr. M. Kleidorfer Institut für Infrastruktur, Arbeitsbereich für Umwelttechnik, Universität Innsbruck, Technikerstraße 13, 6020 Innsbruck, Österreich

yannick.back@uibk.ac.at

Dipl.-Geogr. M. Bremer . PD Dr. M. Rutzinger Institut für Geographie, Universität Innsbruck, Innrain 52f, 6020 Innsbruck, Österreich Institut für Interdisziplinäre Gebirgsforschung, Österreichische Akademie der Wissenschaften, Technikerstraße 21a, ICT, 6020 Innsbruck, Österreich ferenzierte Daten, deren Maßstabsebenen und Verfügbarkeit zu geben. Zudem werden Anwendungsbeispiele, wie sie in der Siedlungswasserwirtschaft zur Einbindung von dezentralen Entwässerungssystemen vorkommen, dargestellt und beschrieben. Neben Untersuchungen der Fließrichtungen und Akkumulation des Regenwassers an der Oberfläche werden auch Methoden gezeigt, welche die Planung von grüner und blauer Infrastruktur erleichtern, um existierende Anlagen, vor allem bei Starkniederschlägen, zu entlasten.

Schlüsselwörter Daten · GIS · Fernerkundung · Stadtentwicklung · Stadthydrologie $\cdot$ dezentrale Entwässerung · Modellierung

\section{Applications of Geographic Information Systems in Urban Water Management- Implementing decentralized stormwater techniques to reduce the pressure on the urban drainage system}

Abstract In the course of digitization and data availability, geographic information systems (GIS applications) are becoming increasingly attractive in many areas, including urban water management. Approximately $90 \%$ of the world's digital data has been collected in the last two years alone. The availability of data offers on the one hand a new potential of application possibilities, but on the other hand also confusion due to this excess of information. The aim of this work is to give an overview of georeferenced data, their different scales and accessibilities, as well as how they can be used in water management emphasizing the implementation of decentralized stormwater management. Besides investigations of the flow directions and accumulation of rainwater at the surface, methods are shown which support the planning of green and blue infrastructure to provide additional capacities, especially in case of heavy rainfall events.

Keywords Data - GIS · RemoteSensing · Urban Development · Urban Hydrology · Decentralized Drainage System $\cdot$ Modelling

\section{Einleitung}

Geoinformationssysteme (GIS) sind Informationssysteme zur Erfassung, Bearbeitung, Verwaltung, Organisation, Analyse und Präsentation räumlicher Daten. Sie umfassen die dazu benötigte Hardware, Software, Daten und Anwendungen. Der Software-Bereich gestaltet sich vielseitig. Geoportale oder WebGIS, wie beispielsweise „GeoDaten Burgenland“, „tirisMaps vom Land Tirol“ oder auch „Google Maps“, liefern dem Nutzer Zugriff auf bestimmte vorausgewählte Daten und bieten die Möglichkeit, diese zu analysieren und zu visualisieren. Open-Source-GIS, wie GRASS GIS (GRASS GIS Development Team 2018) oder QGIS (QGIS Development Team 2019) und kommerzielle GISHersteller (z.B. ESRI mit ArcGIS (ESRI 2016) oder MicroImages mit TNTgis (MicroImages 2015)) bieten die Möglichkeit, die erfassten Geodaten mit einer Vielzahl an Tools für raum-zeitliche Analysen in einem geografischen Bezugssystem zu bearbeiten.

Geoinformationen bestehen aus Sachdaten und Geodaten. Grundsätzlich werden Vektor- und Rasterdatenmodelle unterschieden. Vektordaten repräsentieren die Objektgeometrie anhand der Entitäten Punkte, Linien oder Flächen. Rasterdaten bestehen aus einem Gitter von Bildpunkten (Pixel), dem ein Pixelwert (digital number) zu- 
geordnet ist (z.B. digitale Bilder wie Luft- oder Satellitenbilder, eingescannte Karten etc.). Auch eine Konvertierung von Raster- zu Vektordaten und umgekehrt ist möglich. Digitale Geodaten werden auch für die Siedlungswasserwirtschaft erfasst, verwaltet, analysiert und visualisiert. Leitungsinfrastrukturen (Kanalnetze, Wasserversorgungsleitungen) werden in Leitungsinformationssystemen verwaltet. Computergestützte Anwendungen, wie beispielsweise hydrologische oder hydraulische Modelle im Bereich der Wasserversorgung oder der Siedlungsentwässerung, basieren auf digitalen Geodaten und Analysewerkzeugen, die sowohl zur Modellerstellung als auch für die Visualisierung zum Einsatz kommen. Die stetige Weiterentwicklung von Prozessierungsmethoden, Rechenleistung und Verfügbarkeit von digitalen Geodaten ermöglicht es immer detailliertere Datensätze zu erstellen, zu verwalten und $\mathrm{zu}$ analysieren (Sitzenfrei et al. 2014; Tscheikner-Gratl et al. 2015).

Ziel dieser Arbeit ist es, einen Überblick über digitale Geodaten sowie deren Verfügbarkeit, im Hinblick auf die Siedlungsentwässerung, zu geben. Des Weiteren werden ausgewählte Anwendungsbeispiele beschrieben, welche aktuelle Forschungsfragen aus der Siedlungswasserwirtschaft behandeln. In dieser Arbeit wird der Schwerpunkt auf die Einbindung dezentraler Entwässerungssysteme zur Entlastung des städtischen Abwassernetzes gelegt. Mit der stetig steigenden Einwohnerzahl urbaner Gebiete, der einhergehenden Flächenversiegelung und den intensiver werdenden Starkniederschlagsereignissen wächst die Belastung auf die städtischen Entwässerungssysteme und das Überflutungsrisiko steigt (Kleidorfer et al. 2018). Das Intergovernmental Panel on Climate Change (IPCC) prognostiziert eine deutliche Veränderung der Niederschlagsmengen und vermehrte extreme Niederschlagsereignisse, vor allem für Tropenregionen und Regionen in den mittleren Breiten (IPCC 2014). Im Hinblick auf diese Veränderungen sehen sich Städte in naher Zukunft vor großen Herausforderungen, welchen sie mit nachhaltigen Anpassungsmaßnahmen entgegenwirken müssen. Konzepte zur Einbindung von grüner und blauer Infrastruktur sowie eine Umgestaltung von zentralen zu dezentralen Entwässerungssystemen haben sich in Forschung und Praxis bereits etabliert (Magistrat der Stadt Wien
2010; Foster et al. 2011; Jones und Somper 2013; Matzinger et al. 2017; StEB Köln 2017). Im Gegensatz zur zentralen Entwässerungsmethodik leiten dezentrale Entwässerungstechniken das Niederschlagswasser nicht sofort in das Abwassernetz, sondern behandeln es direkt vor Ort. Durch Speicherung, Infiltration und Evapotranspiration kann Niederschlagswasser zurückgehalten und eine Annäherung an einen natürlicheren Wasserkreislauf geschaffen werden. Weiteres kann dieses Wasser lokal wiederverwendet und unter Umständen zeitverzögert dem bestehenden Entwässerungsnetz zugeführt werden. Durch diese Maßnahmen wird das urbane Abwassernetz entlastet und zusätzliche Möglichkeiten für weitere Nutzenfaktoren (sog. „Multi-Benefits“, wie z.B. Ökosystemdienstleistungssteigerung, Förderung der Biodiversität oder Reduktion von städtischen Hitzeinseln) können entstehen (Woods Ballard et al. 2005).

\section{Datenportale und Datengrundlagen}

In Geoinformationssystemen können Messdaten und bestehende Geoinformationsdaten verarbeitet werden. So stellen beispielsweise Bundes-, Länderportale, Services der Europäischen Union sowie kommerzielle Anbieter digitale Geodaten kostenfrei oder kostenpflichtig zur Verfügung. Auf Bundesebene stellt das Online-Portal „www.data. gv.at" für sämtliche Bundesländer eine Auswahl an frei zugänglichen digitalen Daten - u. a. auch Geoinformationsdaten - zu relevanten Themen, wie beispielsweise Überflutungsflächen, zur Verfügung. Das Bundesministerium für Nachhaltigkeit und Tourismus (BMNT) erhebt Geoinformationsdaten aus den Aufgabenbereichen der Ressorts Landwirtschaft, Forstwirtschaft, Umwelt und Wasserwirtschaft. $\mathrm{Zu}$ den vom BMNT betriebenen Geoportalen zählen z.B. Altlasten GIS, eBOD - Digitale Bodenkarte, eHYD - Hydrographischer Dienst, HORA - Hochwasserrisikozonierung Austria, ISDW - Initiative Schutz durch Wald, Lärminfo, EDM GIS - Assistent zur digitalen Verortung von umweltrelevanten Standorten und Anlagen, WISA - Wasser Informationssystem Austria und AgrarGIS (BMNT 2019). Das Bundesamt für Eich- und Vermessungswesen (BEV) stellt über das Portal „bev.gv.at“ kostenpflichtige Geoinformationen wie Orthofotos und digitale
Höhenmodelle bereit. Aber auch die digitale Katastralmappe (DKM) kann hier bezogen werden. Auch auf Bundesländerebene werden GIS-Portale mit Möglichkeit zur Datenbestellung oder Download betrieben. Zusätzlich wird über den Geodatenverbund der Länder "geoland.at" Zugriff auf Geodaten und Services der Länder für unterschiedliche Zwecke ermöglicht. Die Stadt Wien und österreichische Länder bzw. Ämter der Landesregierungen betreiben die frei verfügbare Verwaltungsgrundkarte von Österreich auf dem Server „basemap.at" (2016), basierend auf den Geodaten der Länder und deren Partnern. Europaweite Geodatensätze werden von der Europäischen Umweltagentur (EEA) verwaltet (EEA 2019).

Eine wichtige Grundlage für Geoinformationssysteme sind raumbezogene Daten, welche berührungslos von verschiedenen Fernerkundungssystemen (Plattformen und Sensoren) aufgenommen werden. Fernerkundungsplattformen umfassen

- Satelliten,

- luftgestützte Systeme (Flugzeug oder Helikopter),

- unbemannte Fluggeräte (UAV) und

- mobile und statische Kartierungssysteme (terrestrische Systeme).

Grundsätzlich unterscheiden sich Geodaten je nach Plattform in ihrer Entfernung zum Beobachtungsobjekt und somit auch in ihrer räumlichen und zeitlichen Auflösung (Abb. 1; Toth und Józków 2016). Das Erfassen von Daten tragbarer mobiler Sensoren (z.B. Smartphones) wird als „Crowdsensing“ bezeichnet (See et al. 2016).

Je nach Fernerkundungsplattform kann eine definierte Konfiguration von Abdeckung und Auflösung (räumlich, zeitlich, radiometrisch und spektral) zur Erfassung der Erdoberfläche erreicht werden. Ständige Weiterentwicklungen im Bereich der Sensorik und Computertechnik senken die Ausstattungskosten, führen $\mathrm{zu}$ leichteren SensorPlattformkonfigurationen und höheren Auflösungen. Auch können Plattformen mit mehreren Sensoren ausgestattet werden, wodurch redundante oder sich ergänzende Sensoren, wie beispielsweise mehrere Kameras und/oder topografische LiDAR (Light Detection and Ranging)-Sensoren, kombiniert werden (Toth und und Józków 2016). Die Maßstabsebenen und folglich auch die Anwendungsbereiche einzelner Plattformen werden in den nächsten Un- 


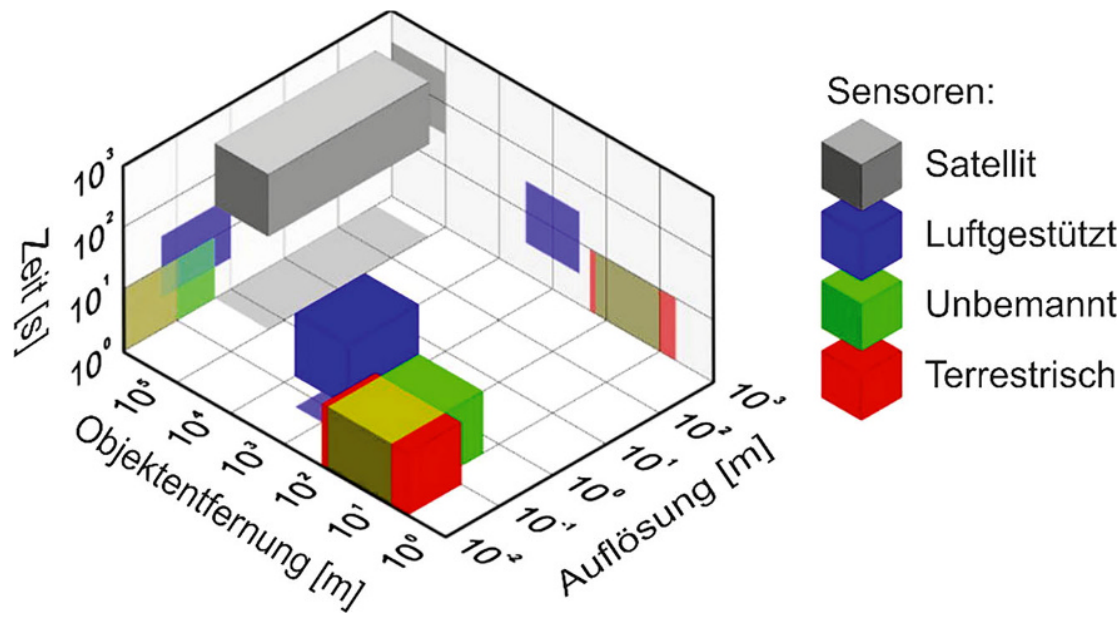

Abb. 1 Räumlicheund zeitliche Auflösung von raumbezogenen Daten. (Nach Toth und Józków 2016)

terkapiteln näher beschrieben und in Tab. 1 zusammengefasst dargestellt.

\subsection{Satellitendaten}

Klassische Anwendungsgebiete der Satellitenfernerkundung sind die Wetterund Klimaforschung sowie die Landbedeckungskartierung. Heute gibt es eine enorme Vielzahl an Zugriffswegen zu unterschiedlichen Datenanbietern (DLR 2019). Satellitenprodukte unterscheiden sich grundsätzlich in ihrer Konstellation, Auflösung und in der zeitlichen Abfolge der Überflüge. Landsat-Satelliten begannen die Erdbeobachtung 1972 und stellen mit über 40 Jahren das längste immer noch laufende Erdbeobachtungsprogramm der Welt dar (Lula et al. 2012). Im Jahr 1986 folgte SPOT-1 und 1999 IKONOS. Seit 1962 registriert das „United Nations Of- fice for Outer Space Affairs" (UNOOSA) alle Objekte, welche ins Weltall befördert werden und zählt zurzeit mehr als 8000 Objekte, von denen jedoch nur ein kleiner Teil zur Erdbeobachtungszwecken dient. Dominiert wird das Register von Kommunikationssatelliten (UNOOSA 2019).

Während früher v.a. einzelne Satellitenmissionen durchgeführt wurden, wird heute v.a. Satelliten-Konstellation betrieben, um die Frequenz der Überflüge und somit die zeitliche Auflösung zu erhöhen. Die ersten Konstellationen wurden von Landsat-, SPOT- und GeoEye/WorldView-Satelliten aufgebaut (Toth und Józków 2016). Das Copernicus-Programm der ESA umfasst 6 Sentinel-Missionen mit verschiedenen Sensorkonfigurationen wie multispektrale Sensoren, Radar-Sensoren, Altimeter etc. Um die großen Daten- mengen verwalten und direkt den individuellen NutzerInnengruppen zur Verfügung stellen zu können, wurden auf Initiative der ESA die TEPs (Thematic Exploitation Platforms), virtuelle Plattformen zur Verbreitung und Benutzung von Erdbeobachtungsdaten, aufgebaut. Die TU Wien, die ZAMG (Zentralanstalt für Meteorologie und Geodynamik) und Firmenpartner betreiben das EODC (Earth Observation Data Centre for Water Resources Monitoring) und stellen ihrerseits große Erdbeobachtungsdatenmengen mit Prozessierungsmöglichkeiten an einem High-Performance-Cluster (HPC) zur Verfügung. Eine sehr spezifische Satelliten-Konstellation ergibt sich, wenn Satelliten in einem Tandem- oder Dualmodus operieren (Dunn et al. 2003). Das argentinische Unternehmen Satellogic bedient sich dieser Technik und hat sich zum Ziel gesetzt, etwa 90 Satelliten in den Orbit zu befördern, um den gesamten Planeten mit einer Auflösung von einem Meter für Multispektralkameras und $30 \mathrm{~m}$ für Hyperspektralkameras wöchentlich abzudecken. Des Weiteren soll es möglich sein, spezifische Orte bis zu sechs Mal am Tag überfliegen zu können (Satellogic 2019).

\subsection{Luftgestützte Daten}

Luftgestützte Daten werden entweder von Flugzeug- oder Helikopterüberflügen erfasst. Durch die geringere Distanz zwischen Sensor und Erdoberfläche kann eine höhere räumliche Auflösung im Vergleich zu Satellitendaten erreicht werden. Die räumliche Auflösung von Sensoren multispektraler Satellitenmissionen verbessert sich jedoch ständig

Tab. 1 Anwendbarkeit und Eigenschaften von raumbezogenen Daten unterschiedlicher Herkunft. (Angepasst nach Toth und Józków 2016)

\begin{tabular}{|c|c|c|c|c|c|}
\hline Anwendbarkeit & Satelliten & Luftgestützt & Unbemannt (UAV) & Mobil/statisch & Crowdsensing \\
\hline Manövrierfähigkeit & Eingeschränkt & Moderat & Hoch & Eingeschränkt & Hoch \\
\hline Beobachtungsraum & Weltweit & Regional & Lokal & Lokal & Lokal \\
\hline Sensoren & MS/HSI/SAR & MS/HSI/LiDAR/SAR & MS (LiDAR/HSI) & MS/LiDAR (HSI) & MS/Video \\
\hline Umgebung & Draußen & Draußen & Draußen/Drinnen & Draußen/Drinnen & Draußen/Drinnen \\
\hline Maßstab & Klein & Klein/medium & Medium/groß & Medium/groß & Medium/groß \\
\hline Bodenabdeckung & Groß - $10 \mathrm{~km}$ & Medium - $1 \mathrm{~km}$ & Klein $-100 \mathrm{~m}$ & Klein - $50 \mathrm{~m}$ & Klein - 10m \\
\hline Sichtfeld & Eng & Weit & Weit/super weit & Weit/super weit & Weit/super weit \\
\hline Wiederholung & Tag & Stunde & Minute & Minute & Minute/Sekunde \\
\hline Räumliche Auflösung & $0,30-300 m$ & $5-25 \mathrm{~cm}$ & $1-5 \mathrm{~cm}$ & $1-5 \mathrm{~cm}$ & $1-5 \mathrm{~cm}$ \\
\hline Räumliche Genauigkeit & $1-3 m$ & $5-10 \mathrm{~cm}$ & $1-25 \mathrm{~cm}$ & $3-50 \mathrm{~cm}$ & $1-2 m$ \\
\hline Einsetzbarkeit & Schwer & Komplex & Einfach & Moderat & Einfach \\
\hline Art der Beobachtung & Vertikal/geneigt & Vertikal/geneigt & Vertikal/geneigt $/ 360^{\circ}$ & Geneigt $/ 360^{\circ}$ & Geneigt \\
\hline Betriebsrisiko & Moderat & Hoch & Niedrig & Moderat & Keines \\
\hline Kosten & $€ € € € €$ & $€ € €$ & $€$ & $€ €$ & Keine \\
\hline
\end{tabular}


und erreicht im zivilen Bereich bis $\mathrm{zu}$ $30 \mathrm{~cm}$ bei bis zu täglichen Wiederholraten. Die topografische LiDAR-Technologie kommt auf tragbaren, statischen und mobilen terrestrischen, luftgestützten und satellitenbasierten Plattformen zum Einsatz. Bei time-of-flight LiDARSystemen wird ein Laserpuls ausgesendet, welcher auf der Erdoberfläche reflektiert und wieder empfangen wird. Über die Messung der Laufzeit des Laserpulses, welcher sich mit annähernd Lichtgeschwindigkeit fortbewegt, wird die Entfernung zum Objekt bestimmt. Im Gegensatz zum Laserprofiling wird beim Laserscanning der Laserpuls von einem z.B. rotierenden oder oszillierenden Spiegel abgelenkt und somit ein Flugstreifen mit einer definierten Breite abgescannt. Aufgrund der langsameren Fluggeschwindigkeit beim helikoptergestützten Laserscanning können höhere Punktdichten als bei flugzeuggestützten Überflügen erreicht werden, was v.a. bei linienhaften bzw. Korridoranwendungen von Vorteil sein kann (Höfle und Rutzinger 2011; Toth und Józków 2016). Die Entwicklung von leichteren Sensoren und Entwicklung von leistungsfähigeren UAV-Plattformen lassen es zu, dass nun auch unbemannte Fluggeräte mit Laserscannern ausgestattet werden können (Wallace et al. 2012; Bremer et al. 2019). Weitere Methoden, luftgestützte Daten zu erfassen, bieten Fotogrammetrie, multispektrale oder hyperspektrale Fernerkundung oder Radar-Systeme.

\subsection{Daten von unbemannten Fluggeräten}

Unbemannte Fluggeräte (engl. Unmanned Aerial Vehicle - UAV, Remotely Piloted Aircraft System - RPAS), in den Medien wird auch häufig der militärische Begriff „Drohnen“ verwendet, operieren entweder eigenständig oder werden aus der Ferne gesteuert. UAVs werden sowohl militärisch als auch kommerziell eingesetzt. Die rechtlichen Grundlagen zum Betrieb von unbemannten Luftfahrzeugen finden sich in den $\$ \S 24 c-241$ des Luftfahrtgesetzes (LFG 1957; idgF.). Die zuständige Behörde zur Erteilung der luftfahrtrechtlichen Bewilligung in Österreich ist die Austro Control GmbH. Durch die hohe Flexibilität für hochauflösende fernerkundliche Aufnahmen im Nahbereich finden UAVs immer häufiger Anwendung im wissenschaftlichen und kommerziellen Bereich. UAV-Befliegun- gen dürfen in Österreich auf Sicht, im Umkreis von $500 \mathrm{~m}$ und bis $\mathrm{zu}$ einer Höhe von $150 \mathrm{~m}$ über Grund durchgeführt werden (LFG 1957; idgF; Pajares 2015). Ein Anwendungsbeispiel in der Siedlungswasserwirtschaft ist die Erfassung von Kanaleinläufen mittels UAVBildern mit einer räumlichen Auflösung von ca. $3 \mathrm{~cm} /$ Pixel (Moy de Vitry et al. 2018).

\subsection{Mobile und statische Kartierungssysteme}

Mobile Kartierungssysteme (engl. Mobile Mapping System - MMS) sind Sensoren, die auf z.B. Autos, Zügen oder Schiffen montiert werden, um Geoinformationen über z. B. Transportwege zu generieren (Ellum und El-Sheimy 2002; Puente et al. 2013; Toth und Józków 2016). Ein bekannter Anwendungsbereich dieser Technik ist beispielsweise „Street View“ von Google. Zur Generierung dieser Daten nutzt Google eine R7-Panoramakamera, montiert auf Autos, Fahrrädern oder auch Schneemobilen (Anguelov et al. 2010). Zu den statischen Kartierungssystemen zählt zudem das bereits im Kapitel Luftgestützte Daten erwähnte terrestrische Laserscanning, aber auch terrestrische fotogrammetrische Systeme, Webcams sowie time laps-Kameras.

\subsection{Crowdsensing}

Durch die Entwicklung sehr leichter und günstiger Sensoren, wie z. B. Smartphone mit Kamera, GNSS (Globales Navigationssatellitensystem) und Lagesensor, kann mittlerweile jede Person eine potenzielle Erkundungsplattform sein. Crowdsensing beschreibt den Vorgang, mithilfe mobiler Geräte generierte Daten zu erfassen und zu analysieren. Interessant wird diese Technik besonders dann, wenn eine große Gruppe von Personen mit mobilen Geräten Daten zu einem bestimmten Thema oder Ereignis (z. B. Bilder von Überflutungsbereichen während eines extremen Niederschlagereignisses) erfasst und verschickt (vgl. See et al. 2016). In der Siedlungswasserwirtschaft kann via Crowdsensing beispielsweise eine Art Validierungsdatensatz für hydrodynamische Modelle bei Extremereignissen entstehen (vgl. Griesbaum et al. 2017).

\section{Anwendungsbeispiele in der Siedlungswasserwirtschaft}

In diesem Kapitel sollen Möglichkeiten und Anwendungsbeispiele von GIS in der Siedlungswasserwirtschaft erläutert werden, die teilweise aus der Literatur stammen, aber auch Gegenstand aktueller Forschungsprojekte sind. Da der Einsatz von GIS-Anwendungen in der Siedlungswasserwirtschaft (SWW) sehr weit verbreitet und vielfältig ist und kontinuierlich zunimmt, kann hier nur ein kleiner Ausschnitt behandelt werden. Der Fokus wird dabei auf die Einbindung dezentraler Lösungsansätze zur Entlastung der urbanen Entwässerungssysteme gelegt. Als Untersuchungsgebiet wurde die Stadt Innsbruck gewählt. In den folgenden Abbildungen wird zur besseren Veranschaulichung nur ein bestimmter Bereich im Gebiet Amras-Rossau im Südosten der Stadt dargestellt.

\subsection{Flächenversiegelungsgrad}

Einer der wichtigsten Parameter für die Niederschlag-Abfluss-Modellierung ist der Flächenversiegelungsgrad (auch Befestigungsgrad), welcher in direktem Zusammenhang mit dem Abfluss von Regenwasser steht (Gujer 2007). Traditionell wird die Flächenversiegelung schon lange durch die Auswertung von Orthofotos ermittelt. Zumeist folgt dies einer manuellen Bearbeitung, bei der befestigte Flächen (Dächer, Straßen etc.) nachgezeichnet werden. Ein neuer Ansatz besteht darin, den Flächenversiegelungsgrad über den Normalized Difference Vegetation Index (NDVI) abzuschätzen, welcher sich über die Reflexionswerte der Lichtwellen im nahen Infrarotbereich (NIR) und dem roten sichtbaren Bereich (Rot), wie folgt errechnen lässt:

$$
\mathrm{NDVI}=\frac{(\mathrm{NIR}-\mathrm{Rot})}{(\mathrm{NIR}+\mathrm{Rot})} \text {. }
$$

In diesem Beispiel wurde der NDVI aus dem CIR (Color-Infrared)-Falschfarbenbild (bereitgestellt vom Land Tirol 2019) errechnet. Falschfarbenbilder eignen sich zur Untersuchung von Vegetationsänderungen oder Bestimmungen von Pflanzenarten. Aus dem NDVI werden vegetative und nicht vegetative Flächen ermittelt. Es ergibt sich ein Wertebereich von-1 bis 1 . Negative Werte bezeichnen Wasserflächen, Werte zwischen 0 und 0,2 entsprechen nicht vegetativen Flächen und darüber 


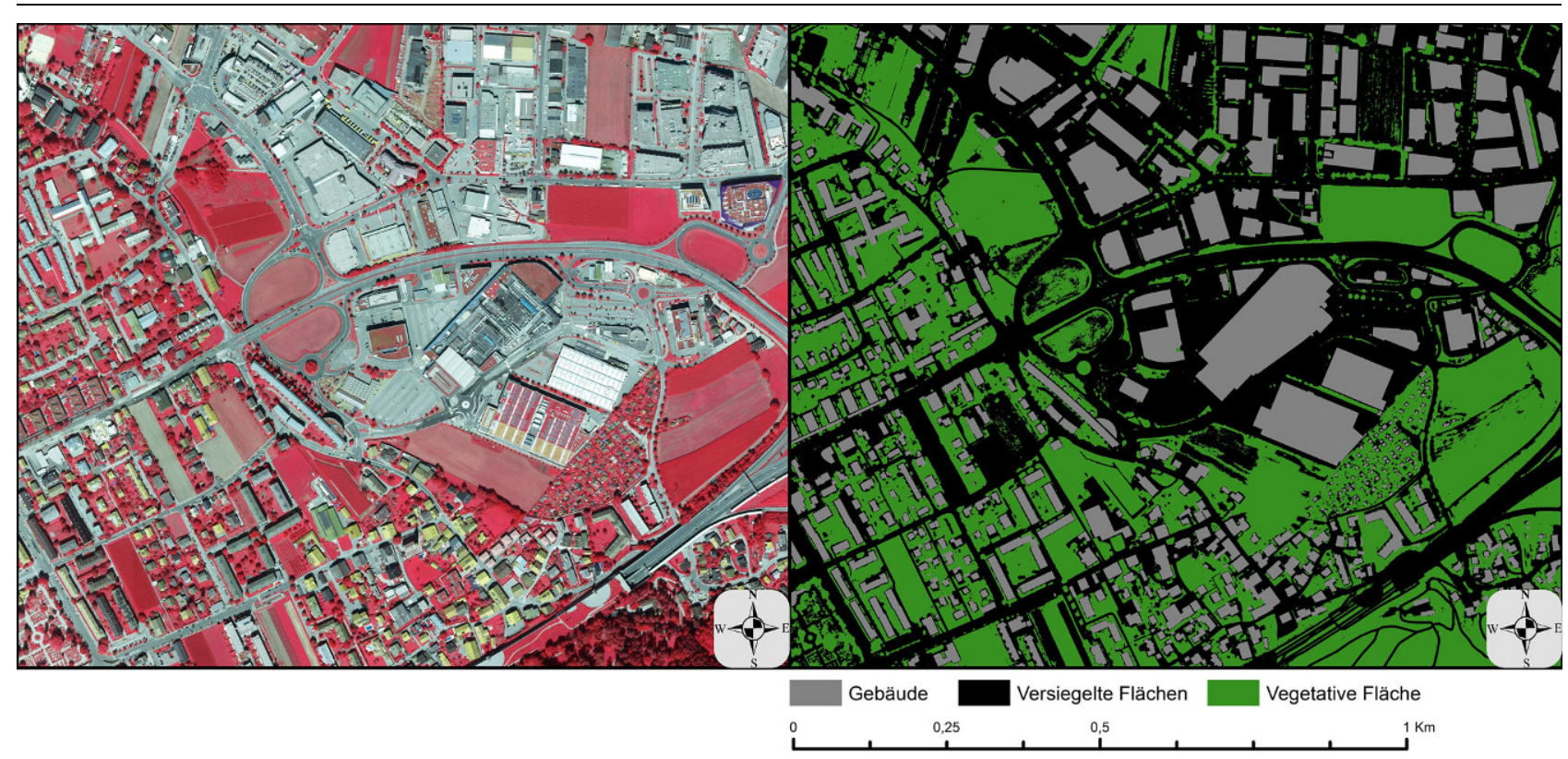

Abb. 2 GIS-Analyse des Flächenversiegelungsgrads am Beispiel Amras-Rossau (Innsbruck)

liegende Werte stehen für vegetative Flächen (Bannari et al. 1995). Werte nahe 1 weisen auf eine hohe Chlorophyllaktivität hin. Auf Grundlage dieser Unterteilung wurde eine Kategorisierung der berechneten NDVI-Werte vorgenommen. Da für diese Berechnungen ausschließlich die Unterscheidung in vegetative und versiegelte Flächen von Relevanz war, wurde ein Schwellenwert bei 0,2 gesetzt. Alle darunterliegenden Werte wurden als versiegelte Flächen und alle höheren Werte als vegetative Flächen gekennzeichnet. Anschließend wurden die Gebäude und Fließgewässer herausgerechnet, sodass sich eine Annäherung an den Flächenversiegelungsgrad (Abb. 2 rechts) ergab. Klassifikationsalgorithmen wie z.B. jene des maschinellen Lernens (z.B. Neuronale Netze) können Landnutzungsklassen wie Gebäude oder Grünflächen mithilfe von Trainingsdaten automatisiert ableiten (siehe z. B. Maggiori et al. 2017; Marmanis et al. 2018).

\subsection{Abflussakkumulation und Fließrichtungen}

In urbanen Gebieten sind Fließrichtung und Akkumulation des Abflusses wichtige Parameter zur Analyse von Überflutungsrisiken. Gängige GIS-Lösungen stellen Werkzeuge zur hydrologischen Modellierung zur Verfügung. Diese beinhalten Berechnungen der Fließlänge, Fließdistanz, Fließrichtung, Abflussakkumulation, Wassereinzugs- gebiete etc. In der Regel werden diese Berechnungen nicht für Analysen in urbanen Gebieten durchgeführt, können aber die Planung von Anlagen zur dezentralen Niederschlagsbehandlung, wie z. B. urbane Feuchtbiotope und natürliche Retentionsbecken, durch die Analyse der natürlichen Geländeform unterstützen. Die Fließrichtung wurde auf der Basis eines Digitalen Gelände-Modells (DGM) (bereitgestellt vom Land Tirol 2019) mit einer Auflösung von $1 \mathrm{~m}$ berechnet. Die weitere Berechnung der Akkumulation beinhaltet als Grundlage dasselbe DGM und zusätzlich die Informationen der Berechnung der Fließrichtung. Hierbei wird jedoch kein echtes Überflutungsvolumen berücksichtigt, sondern anhand der Geländeform ausschließlich die Fließbewegung analysiert. Abb. 3 links veranschaulicht die Oberflächenhöhe und gibt Aufschluss über mögliche Senken und potenzielle Überflutungsflächen. Abb. 3 rechts stellt die Akkumulation spezifisch für versiegelte Flächen dar. Vor allem im tiefer gelegenen Bereich um das Einkaufszentrum DEZ lässt sich eine verstärkte Akkumulation feststellen. Bei näherer Betrachtung lässt sich, bedingt durch die Straßenneigung, eine Akkumulation an den Straßenrändern erkennen. Kombiniert mit der Oberflächentopografie lassen sich somit auch Fließrichtungen abschätzen. Wichtig bei der Interpretation ist die Berücksichtigung der Genauigkeit und die Richtigkeit der Gelände- und Objekt- repräsentation in digitalen Geländeund Oberflächenmodellen. So können Objekte wie Zäune oder Vegetation den Abfluss in Modellen fälschlicherweise umlenken oder Durchflüsse, die in den Modellen nicht abgebildet sind, zu fehlerhaften Modellierungsergebnissen führen.

Abb. 4 links beinhaltet die Akkumulation für das gesamte Geländemodell. Abb. 4 rechts weist neben der Überflutungsverteilung auch die Überflutungshöhe, erstellt mit dem Modell RUFIDAM - Rapid Urban Flood Innundation and Damage Assessment Model (Jamali et al. 2018), aus. Dieses numerische Modell entstammt einer Arbeitsgruppe der Monash Universität in Melbourne, Australien. Basierend auf eindimensionalen hydrodynamischen Modellen (SWMM oder MOUSE) und GIS-Technologien, können Ausbreitung, Tiefe und Schaden einer urbanen Überflutung schneller abgeschätzt werden. Auch für diese Berechnungen lieferte ein DGM mit einer Auflösung von einem Meter die Basis (Jamali et al. 2018). Während bei der Akkumulation nur die Topografie einfließt, berücksichtigt das Modell RUFIDAM die Überflutungsvolumina aus dem überlasteten Kanalnetz und die anschließende Verteilung des Regenwassers an der Oberfläche über deren Topografie. 


\section{Originalarbeit}

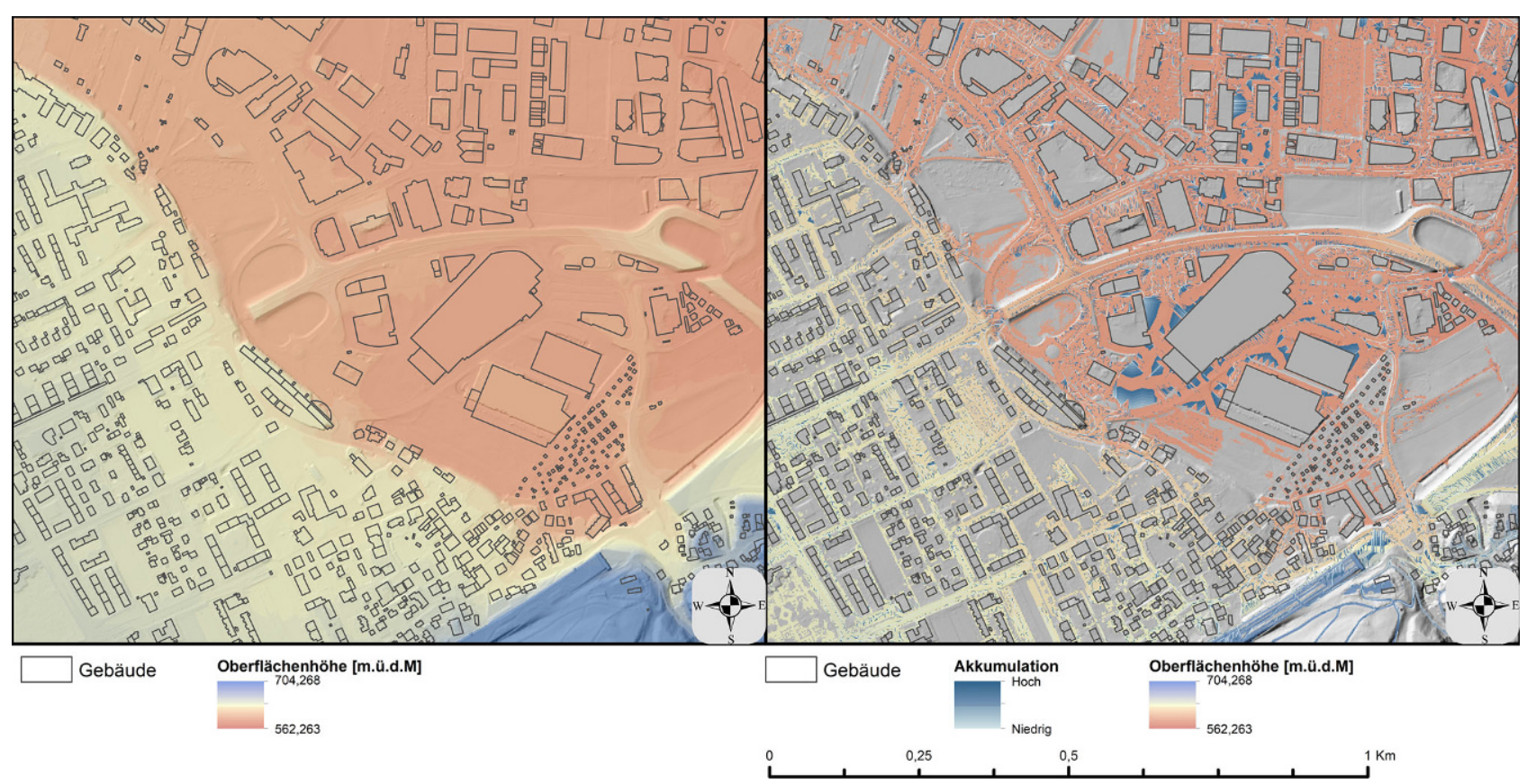

Abb. 3 Darstellung des Geländemodells und Analyse der Oberflächenhydrologie zur Abschätzung der Fließwege

3.3 Strukturtypenanalyse zur Einbindung dezentraler Entwässerungssysteme

Bei der Implementierung dezentraler Infrastruktur hat sich gezeigt, dass für die Realisierbarkeit die Ermittlung der Siedlungsstruktur von großer Be- deutung ist (Simperler et al. 2018). Je nach Strukturtyp können die unterschiedlichen dezentralen Maßnahmen als „empfohlen“, „nicht empfohlen“, „individuelle Beurteilung erforderlich“ oder „zulässig“ eingestuft werden. Zur Erstellung der Siedlungsstrukturtypen für die Stadt Innsbruck wurde die Vorgehensweise nach Simperler et al. (2018) herangezogen. Auf Basis eines Orthofotos (Jahr 2018) mit einer Bodenauflösung von $20 \mathrm{~cm}$ (bereitgestellt vom Land Tirol 2019), wurden nach Simperler et al. (2018) 13 verschiedene Strukturtypen bestimmt: Wohngebiet mit geringer Versiegelung

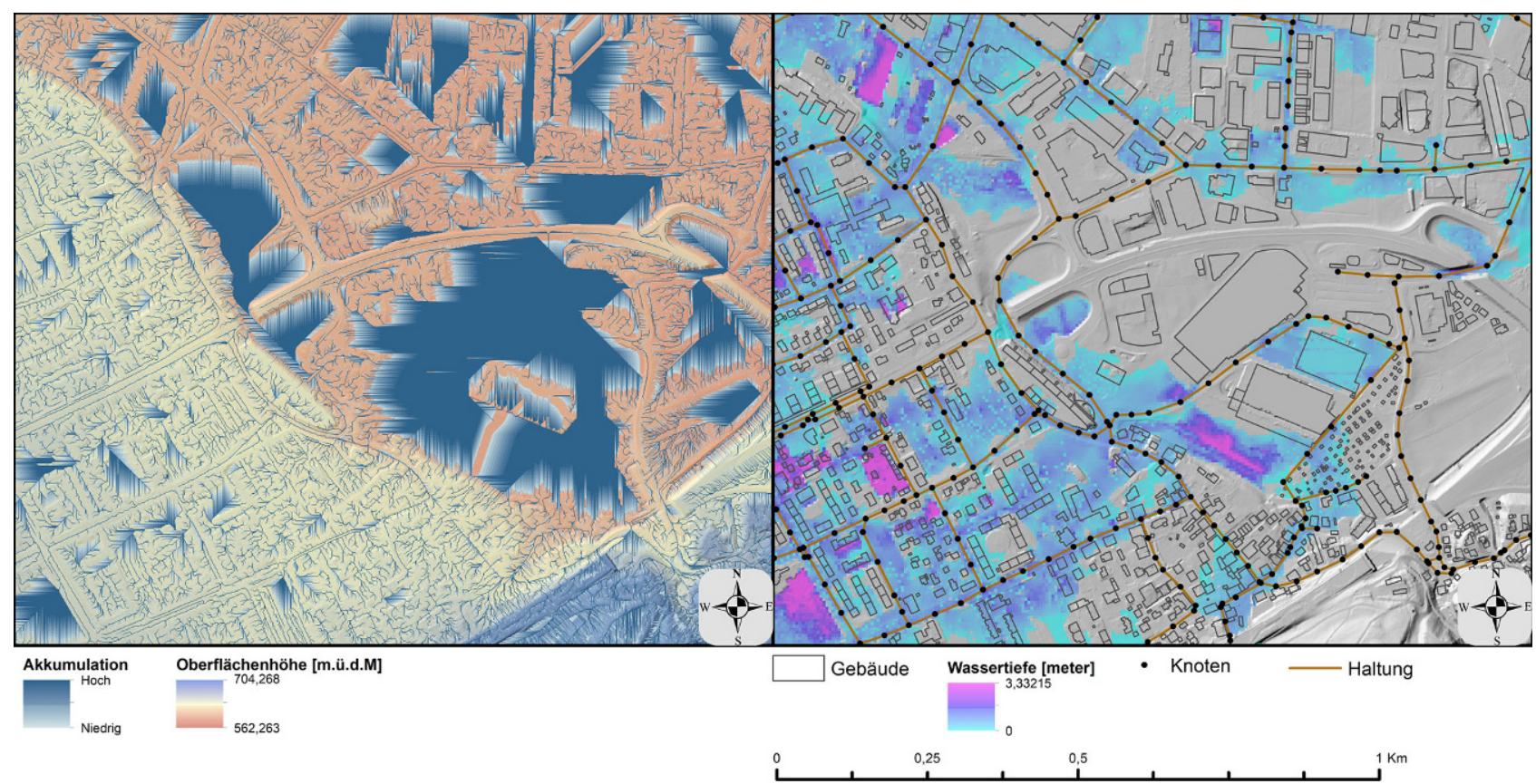

Abb. 4 Darstellung der Akkumulation aus dem Hydrology Tool und der Überflutungsverteilung aus dem Modell RUFIDAM. (Jamali et al. 2018) 


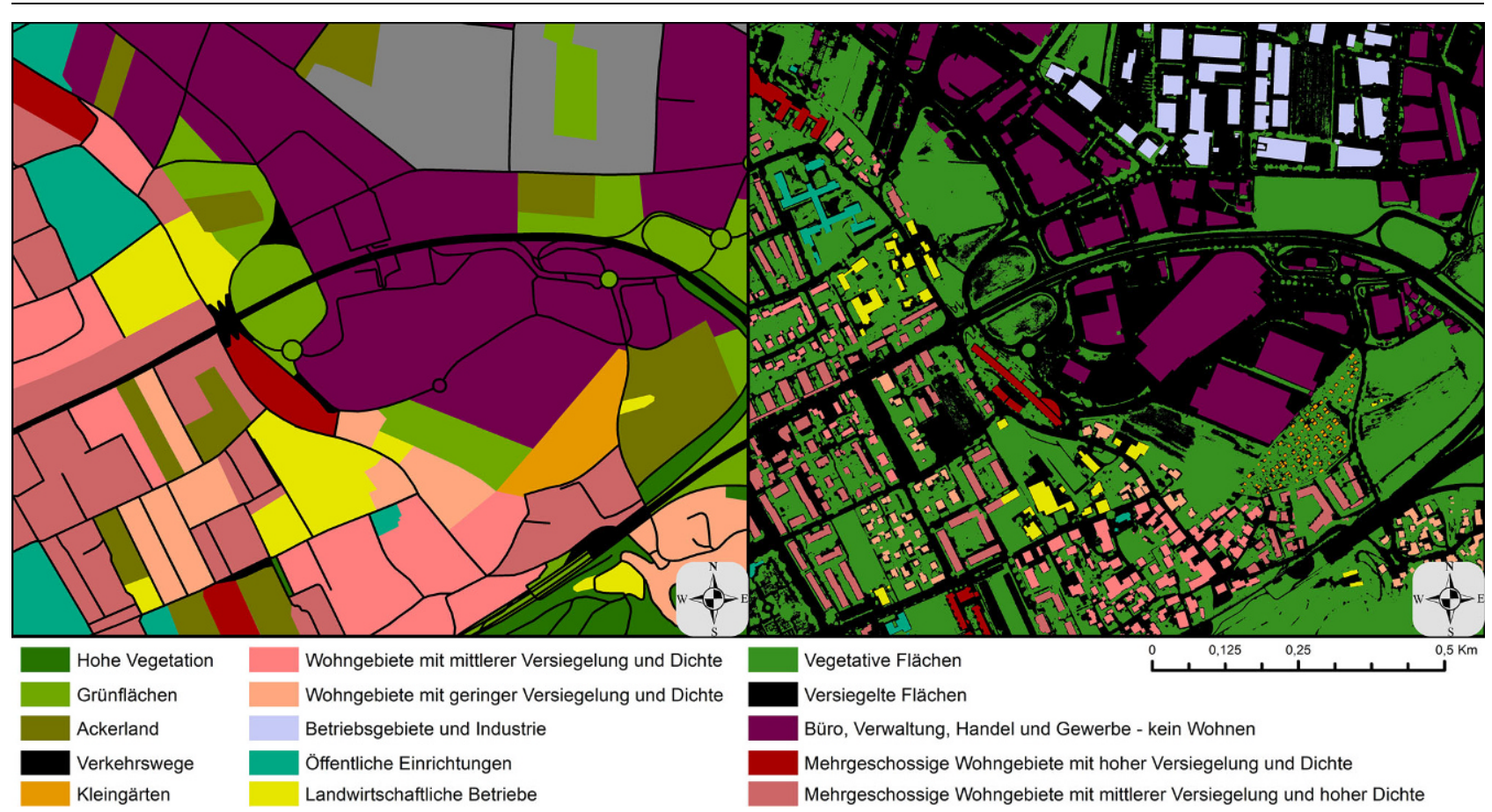

Abb. 5 Siedlungsstrukturtypenanalyse. (Nach Simperler et al. 2018)

und Dichte/Wohngebiet mit mittlerer Versiegelung und Dichte/Kerngebiet mit geringer Versiegelung und Dichte/ Kerngebiet mit mittlerer Versiegelung und Dichte/Mehrgeschossige Wohngebiete mit mittlerer Versiegelung und hoher Dichte/Mehrgeschossige Kerngebiete mit mittlerer Versiegelung und hoher Dichte/Mehrgeschossige Wohngebiete mit hoher Versiegelung und Dichte/Bebauung mit hoher Versiegelung, Dichte und Mischnutzung, Stadtzentrum - Innenstadtbebauung/ Öffentliche Einrichtungen/Büro, Verwaltung, Handel und Gewerbe, kein Wohnen/Betriebsgebiete und Indus-
trie/Dörfliche Gebiete mit landwirtschaftlichen Betrieben/Kleingärten. Fünf weitere Kategorien (Verkehrswege, Gewässerlauf, Hohe Vegetation, Grünflächen und Ackerland) wurden hinzugefügt, um eine vollständig bedeckte Karte generieren zu können. Abb. 5 links zeigt die Siedlungsstrukturtypen

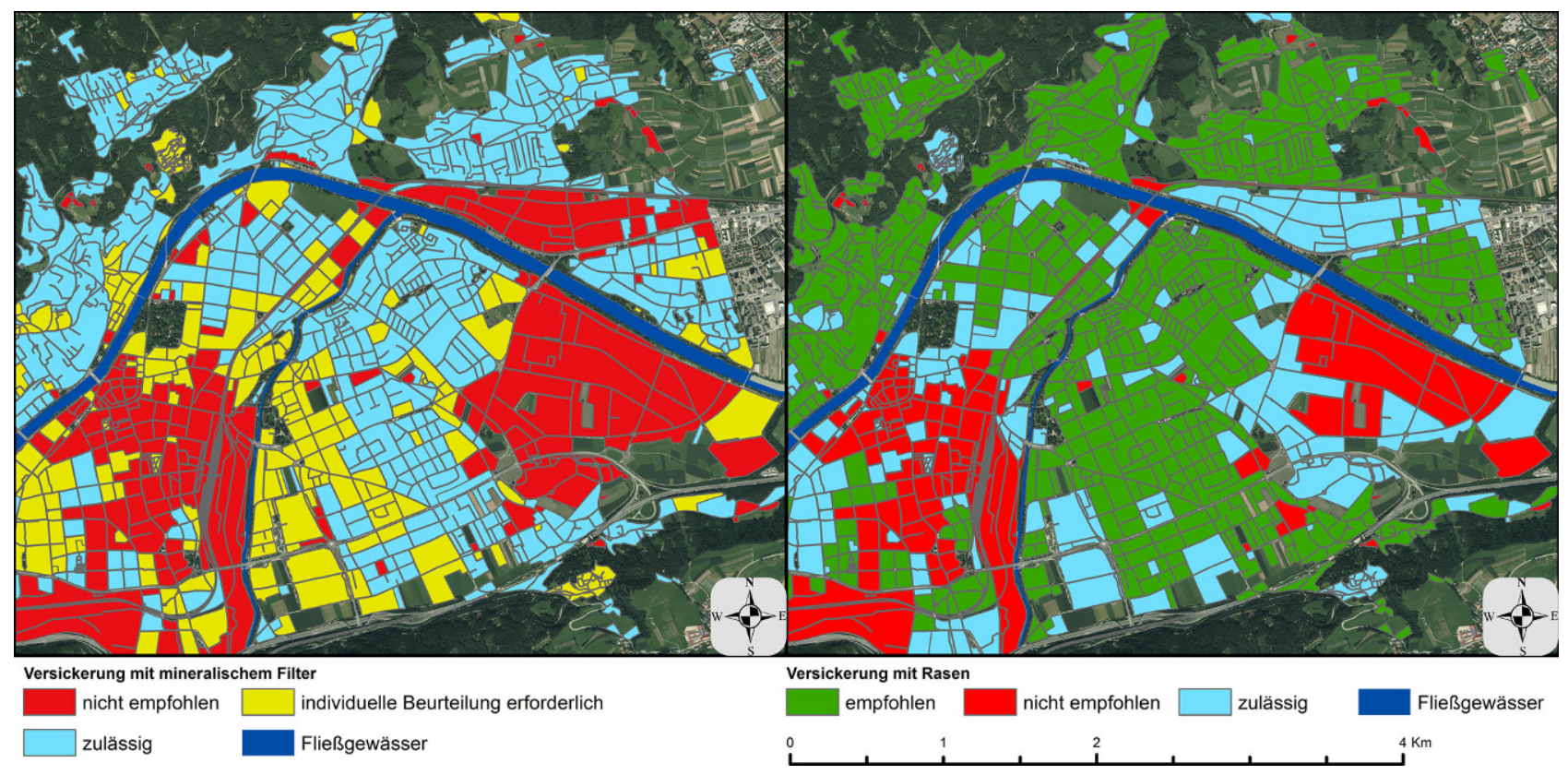

Abb. 6 Empfehlungen zur Implementierung von Versickerungsanlagen mit mineralischem Filter (links) und mit Rasen (rechts) 


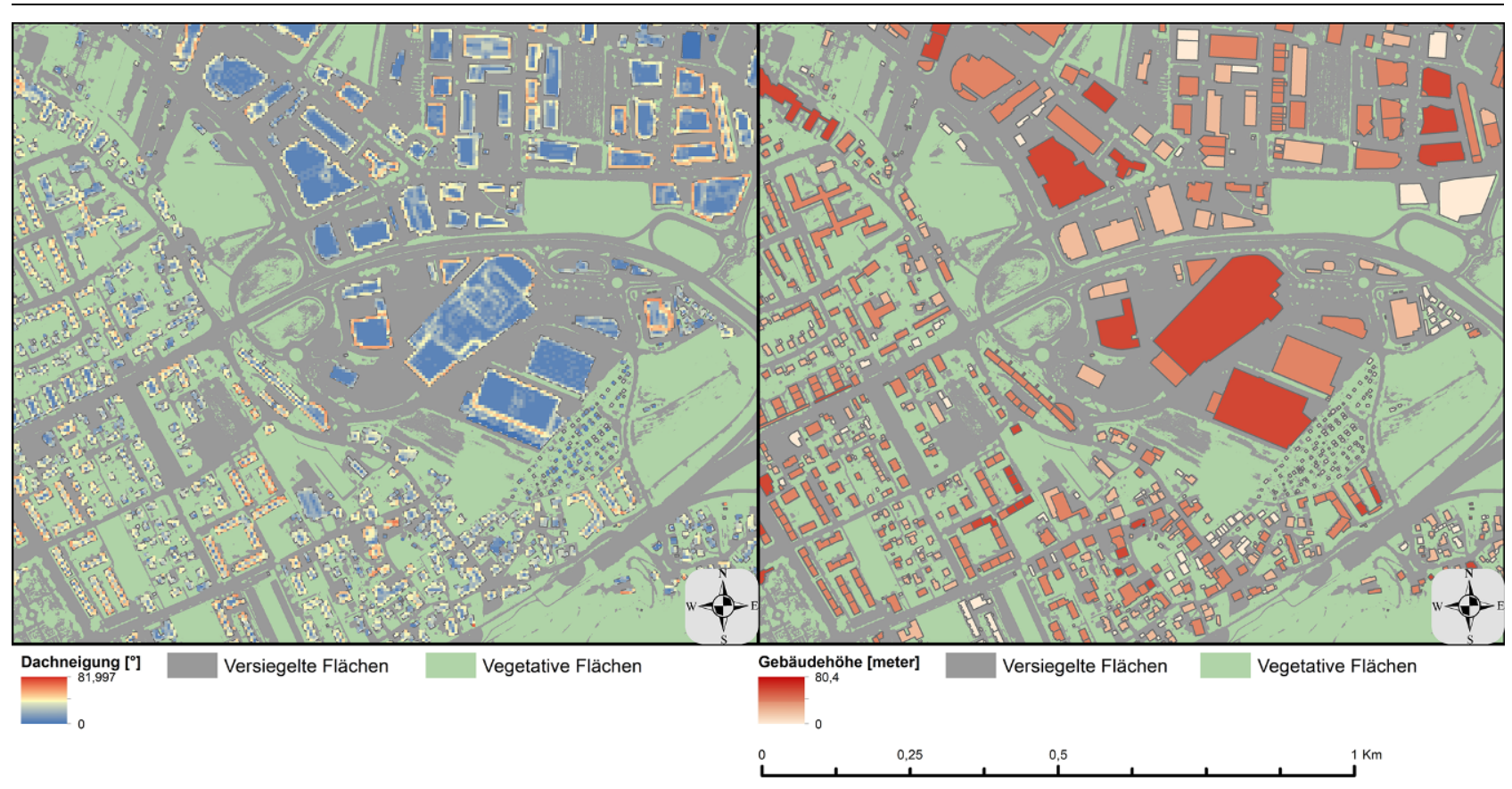

Abb. 7 Dachneigungen und Gebäudehöhen zur Implementierung von Gründächern oder steuerbaren Regentonnen

für das Gebiet Amras-Rossau der Stadt Innsbruck. In weiterer Folge wurden diese auf Gebäudeebene heruntergerechnet und anstelle der Kategorien Verkehrswege, Hohe Vegetation, Vegetation und Ackerland wurden die Ergebnisse aus der Berechnung des NDVI hinterlegt (siehe Abb. 5). Trotz der Berechnung auf Gebäudeebene sind jedoch keine Zusatzinformationen entstanden. Um die Strukturtypen konkret für einzelne Gebäude zu erhalten, wären vertiefte bauliche Informationen (z.B. Gebäudenutzung) nötig.

Die verschiedenen Siedlungsstrukturtypen beinhalten eine Tabelle $\mathrm{zu}$ möglichen Techniken der Niederschlagsbehandlung. In Abb. 6 werden exemplarisch zwei mögliche Arten der Niederschlagsbehandlung (Versickerung mit mineralischem Filter und Versickerung mit Rasen) in Abhängigkeit von den Strukturtypen dargestellt. Dadurch können Empfehlungen zur örtlichen Behandlung des Niederschlagswassers durch Versickerung mit mineralischem Filter oder mit Rasen gegeben werden. Ein Ausschlussverfahren bestimmter dezentraler Maßnahmen an gewissen Standorten wird somit ermöglicht.

Eine zusätzliche Überlagerung dieser Karten mit Sensitivitätskarten aus hydrodynamischen Modellsimulationen, welche die Vorteile der räumlichen LID (Low Impact Development)-Verteilung in Hinblick auf das Abwassernetz
(Zischg et al. 2018) oder der Reduktion von Hitzeinseln (Coutts und Harris 2012) zeigen, ermöglicht eine umfassende Nutzung der Vorteile dezentraler Entwässerungssysteme.

\subsection{Dachflächen als Potenzial für Gründächer oder steuerbare Regentonnen}

Die Neigung der Dachflächen spielt eine wichtige Rolle bei einer möglichen Begrünung sowie bei einer Installation von Solarkollektoren zur Gewinnung von Sonnenenergie. Letztere benötigt zudem die Information der Exposition der ausgewählten Dachfläche. Diese Informationen lassen sich berechnen und veranschaulichen (z.B. Bremer et al. 2016). Das Land Tirol stellt zur Abfrage des Solarpotenzials ein Geoportal (www.tirolsolar.at) zur Verfügung. Des Weiteren lassen sich auch realistische Potenzialstudien zur Implementierung von Regentonnen durchführen, welche den Regenabfluss der Dachflächen auffangen, speichern und für die Bewässerung von Grünflächen verwendet werden können. Damit kann zum einen wertvolles Trinkwasser eingespart werden und andererseits kann das Kanalsystem durch geeignete Steuerung entlastet werden (siehe z. B. Oberascher et al. 2019). Abb. 7 links veranschaulicht die Neigungen der einzelnen Dachflächen. In Verbindung mit der in Abb. 7 rechts dargestellten Gebäudehöhe lassen sich Aussagen zur nutzeneffektiven Dachbegrünung tätigen. In Bezug auf urbanes Mikroklima könnte die Gebäudehöhe einen wichtigen Aspekt darstellen.

\subsection{Grüne Infrastruktur und urbane Hitzeinseln}

Wie bereits erwähnt findet zurzeit eine Umgestaltung von zentralen zu dezentralen Entwässerungssystemen unter Einbindung grüner und blauer Infrastruktur statt. Durch dieses Umdenken und die Implementierung dezentraler Techniken können bei Starkniederschlagsereignissen die Entwässerungssysteme entlastet werden. Als Zusatzeffekt können jedoch auch Mehrfachnutzungen (Multi-Benefits) auftreten. Dazu gehört auch die Reduktion urbaner Hitzeinseln (EPA 2008; Kleerekoper et al. 2012). Mithilfe des GISbasierten Modells DAnCE4Water, eines Programms zur Simulation urbaner Entwicklungen und der Bewertung der Leistungsfähigkeit von spezifischen Eingriffen des Wassermanagements über einen dynamischen Zeitraum, können auch Analysen zu urbanen Hitzeinseln getätigt werden (Bach et al. 2013; Rauch et al. 2017). Abb. 8 zeigt einen Ausschnitt aus einer solchen Analyse am Beispiel der Stadt Innsbruck. Neben der Oberflächentempe- 

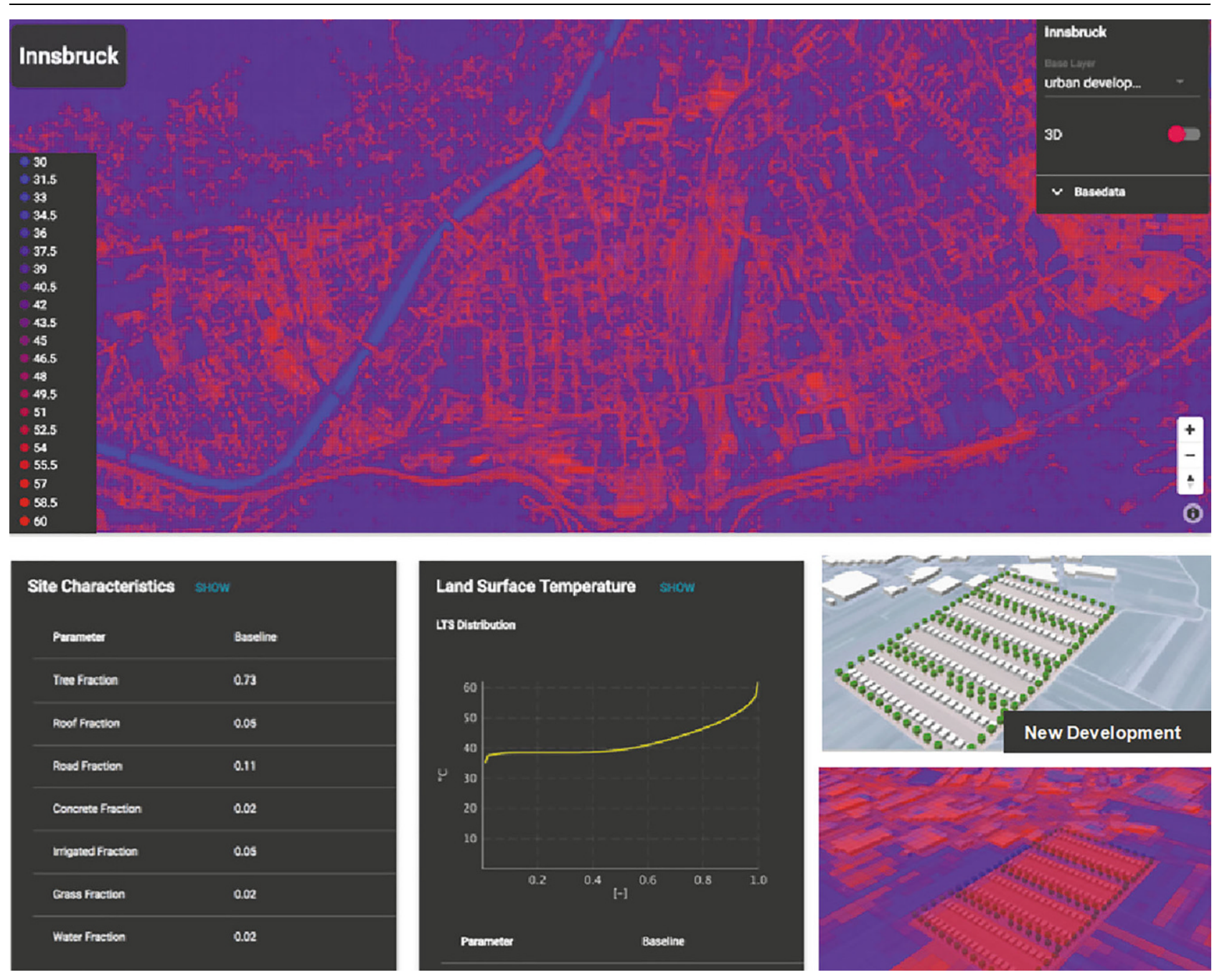

Abb. 8 Analyse von urbanen Hitzeinseln mittels DAnCE4Water

ratur sind auch gewisse Parameter zur Bestimmung der Ortscharakteristik in der Abbildung zu erkennen. Parameter wie beispielsweise Vegetationsanteil, Wasseranteil oder Straßenanteil fließen in die Berechnungen der Oberflächentemperatur mit ein und beeinflussen diese in unterschiedlichem Ausmaß. Auch neue Entwicklungen können mit diesem Programm getestet werden.

Die weitere Erforschung der Mehrfachnutzungen birgt großes Potenzial für die Reduktion von Hitzeinseln als auch für die Siedlungswasserwirtschaft.

\subsection{Automatisierte Erstellung von hydrologisch-hydraulischen Modellen}

Numerische Modelle werden in der Siedlungswasserwirtschaft seit Jahrzehnten angewandt, um mögliche Systemveränderungen (z. B. Klimawandel,
Bevölkerungswachstum und interne Störungen) mittels Szenarien zu untersuchen. Solche „Was-wäre-wennAnalysen“, wie beispielsweise zum Ausfall von Rohrleitungen, ermöglichen einen schnellen und zerstörungsfreien Ansatz, um geplante oder ungeplante Ereignisse $\mathrm{zu}$ bewerten und vorzeitig auf solche reagieren zu können. Da die Erstellung von hydrologisch-hydraulischen Kanalnetzmodellen oft sehr zeitaufwendig ist, entwickelt sich der Trend zur automatisierten Modellerstellung, bei welcher frei zugängliche Daten benutzt werden. Mair et al. (2017) präsentierten einen Algorithmus zur Approximation von Leitungsnetzen oder Ergänzung fehlender Netzteile über Open-Street-Maps. Warsta et al. (2017) stellten einen automatischen Generator (GisToSWMM5) für Teileinzugsgebiete vor, welcher auf frei zugänglichen Daten beruht. Diese Teileinzugsgebiete, samt den ermittelten Parametern wie Neigungen, Fließrichtungen und Flächenkopplungen, können anschließend mit dem Leitungsnetz verknüpft und in SWMM (Rossman 2015) simuliert werden. Durch die Verwendung solcher automatisierter Verfahren können Modellerstellungszeiten deutlich reduziert werden, wobei die Qualität mit manuell erstellten Modellen vergleichbar ist (Warsta et al. 2017).

\section{Schlussfolgerungen und Ausblick}

Die steigende Verfügbarkeit digitaler Geodaten bietet zum einen eine Vielzahl an Analysemöglichkeiten im raumzeitlichen Kontext und für Modellierungen im Bereich Siedlungswasserwirtschaft. Auf der anderen Seite stellt sie den/die Endbenutzer/in vor Schwierigkeiten, für bestimmte Anwendungen geeignete Datensätze auszuwählen. 
Durch Digitalisierungsinitiativen steigt die Verfügbarkeit digitaler Geodaten. Dadurch steigt jedoch auch der Bedarf an entsprechender Software und an Know-how für ein sicheres und einheitliches Datenmanagement, adäquate Analysen und Interpretation von Ergebnissen. Auch in der Siedlungswasserwirtschaft entstehen durch die Digitalisierung neue Potenziale, aber auch neue Herausforderungen. Für die Beschaffung, Verarbeitung und Verwaltung räumlicher Daten sowie die sich mehrenden Einsatzmöglichkeiten von Geoinformationssystemen in der Siedlungswasserwirtschaft stehen verschiedene GIS-Software-Pakete, sowohl Open Source wie auch von kommerziellen Herstellern zur Verfügung. Kostenpflichtige, aber auch kostenfreie Geodaten können über entsprechende Bundes- und Länderportale sowie bei kommerziellen Anbietern bezogen werden. Fernerkundungssysteme liefern wichtige Grundlagendaten für Geoinformationssysteme. Immer leistungsfähigere Sensoren können immer genauere und auch zeitlich höher aufgelöste Datensätze produzieren.

GIS-Anwendungen spielen in der Siedlungswasserwirtschaft eine wichtige Rolle. In diesem Beitrag wurden Beispiele zur Einbindung dezentraler Entwässerungssysteme zur Entlastung des städtischen Abwassernetzes, zur Berechnung des Flächenversiegelungsgrads, der Akkumulation und Fließrichtungen sowie zur Erstellung einer Strukturtypenanalyse zur Einbindung dezentraler Entwässerungssysteme, einer Analyse von Dachflächen als Potenzial für Gründächer oder steuerbare Regentonnen, grüne Infrastruktur zur Minderung urbaner Hitzeinseln und zur automatisierten Erstellung von hydrologisch-hydraulischen Modellen dargestellt. Im Rahmen der voranschreitenden Digitalisierung wächst auch in der Siedlungswasserwirtschaft die Bedeutung raum-zeitlicher Analysen mithilfe von Geoinformationssystemen, um zukunftsorientierte Aufgaben anzugehen und zu lösen.
Förderung Diese Arbeit entstand im Zuge des Projekts CONQUAD (Consequences of adaptation: assessing multibenefits and challenges in the transfer to more resilient and sustainable urban water systems) und wird im Auftrag des Österreichischen Klima- und Energiefonds durchgeführt.

Funding Open access funding provided by University of Innsbruck and Medical University of Innsbruck.

Open Access Dieser Artikel wird unter der Creative Commons Namensnennung 4.0 International Lizenz (http:// creativecommons.org/licenses/by/4. $0 /$ deed.de) veröffentlicht, welche die Nutzung, Vervielfältigung, Bearbeitung, Verbreitung und Wiedergabe in jeglichem Medium und Format erlaubt, sofern Sie den/die ursprünglichen $\mathrm{Au}$ tor(en) und die Quelle ordnungsgemäß nennen, einen Link zur Creative Commons Lizenz beifügen und angeben, ob Änderungen vorgenommen wurden.

Literatur

Anguelov, D., Dulong, C., Filip, D., Frueh, C., Lafon, S., Lyon, R., Ogale, R., Vincent, L. und Weaver, J. (2010): Google street view: capturing the world at street level. Computer (6): 32-38.

Bach, P.M., McCarthy, D.T., Urich, C., Sitzenfrei, R., Kleidorfer, M., Rauch, W. und Deletic, A. (2013): A planning algoritjm for quantifying decentralised water management opportunities in urban environments. Water Science \& Technology (68.8): 1857-1865.

Bannari, A., Morin, D., Bonn, F., und Huete, A R. (1995): A review of vegetation indices. Remote sensing reviews, 13(1-2): 95-120.

BMNT - Bundesministerium Nachhaltigkeit und Tourismus. (2019): GIS-Anwendung des BMNT. Web-Zugriff auf: https://www.bmnt.gv. at/service/geo-informationen.html [Zuletzt besucht am: 09.04.2019].

Bremer, M., Mayr, A., Wichmann, V., Rutzinger, M. und Schmidtner, K. (2016): A new multi scale 3D-GIS-approach for the assessment and dissemination of solar income of digital city models. Computers, Environment and Urban Systems. Vol. 57: 144-154.

Bremer, M., Wichman, V., Rutzinger, M., Zieher, T. und Pfeiffer, J. (2019): Simulating unmanned-aerial-vehicle based laser scanning date for efficient mission planning in complex terrain. International Archives of Photogrammetry, Remote Sensing and Spatial Information Sciences. Enschede, The Netherlands, in press.

Coutts, A. und Harris, R. (2012): Urban Heat Island Report: A multi-scale assessment of urban heating in Melbourne during an extreme heat event: policy approaches for adaptation. Victorian Centre for Climate Change Adaptation Research. School of Geography and Environmental Science. Monash University, Australia. DLR - Deutsches Zentrum für Luft- und Raumfahrt (2019): Satellitendaten. Web-Zugriff auf: https://www.dlr.de/eoc/satellitendaten/ [Zuletzt Besucht am: 07.03.2019]
Dunn, C., Bertiger, W., Bar-Sever, Y., Desai, S., Haines, B., Kuang, D., Franklin, G., Harris, I., Kruizinga, G., Meehan, T., Nadi, S., Nguyen, D., Rogstad, T., Tomas, J.B., Tien, J., Romans, R. Watkins, M., Wu, S.-C., Bettadpur, S. und Kim, J. (2003): Application challenge-instrument of grace-GPS augments gravity measurements. GPS World 14(2): 16-29.

EEA - Europäische Umweltagentur. (2019): Data and maps. Web-Zugriff auf: https://www.eea. europa.eu/de/about-us [Zuletzt Besucht am: 09.04.2019]

Ellum, C., und El-Sheimy, N. (2002): Landbased mobile mapping systems. Photogrammetric engineering and remote sensing, 68(1): 13-17.

EPA-U.S. Environmental Protection Agency (2008): Reducing Urban Heat Islands: Compendium of Strategies.

ESRI-Environmental Systems Research Institute (2016): ArcMap. Web-Zugriff auf: http:// desktop.arcgis.com/de/arcmap/10.3/managedata/raster-and-images/ndvi-function.htm

[Zuletzt Besucht am: 12.03.2019].

Foster, J., Lowe, A. und Winkelman, S. (2011): The Value of Green Infrastructure for Urban Climate Adaptation. The Center for Clean Air Policy. Washington.

GRASS Development Team (2018): Geographic Resources Analysis Support System (GRASS) Software, Version 7.4. Open Source Geospatial Foundation. Web-Zugriff auf: https://grass. osgeo.org [Zuletzt Besucht am: 08.04.2019].

Griesbaum, L., Marx, S. und Höfle, B. (2017): Direct local building inundation depth determination in 3D point clouds generated from user-generated flood images. Natural Hazards and Earth System Sciences. Vol. 17 (7): 1191-1201

Gujer, W. (2007): Urban water management (in German). Springer, Berlin.

Höfle, B. und Rutzinger, M. (2011): Topographic airborne LiDAR in geomorphology: A technolo- gical perspective. Annals of Geomorphology. Vol. 55(2): 1-29.

IPCC, 2014: Climate Change 2014: Synthesis Report. Contribution of Working Groups I, II and III to the Fifth Assessment Report of the Intergovernmental Panel on Climate Change [Core Writing Team, R.K. Pachauri und L.A. Meyer (eds.)]. IPCC, Geneva, Switzerland, 151 pp.

Jamali, B., Löwe, R., Bach, P.M., Urich, C., Arnbjerg-Nielsen, K. und Deletic, A. (2018): A rapid urban flood inundation and damage assessment model. Journal of Hydrology (564): 1085-1098.

Jones, S. and Somper, C. (2013): The role of green infrastructure in climate change adaption in London. The Geographical Journal. Royal Geographical Society (with the Institute of British Geographers). London.

Kleerekoper, L., van Esch, M. und Salcedo, T. (2012): How to make a city climate-proof, addressing the urban heat island effect. Resources, Conservation and Recycling (64): 30-38.

Kleidorfer, M., Tscheikner-Gratl, F. und Vonach, T. (2018): What can we learn from a 500year event? Experiences from urban drainage in Austria. Water Science \& Technology (77.8): 2146-2154.

Land Tirol (2019): Orthofoto Tirol. Abteilung Geoinformation. Bezogen von data.gv.at. Datum des Metadatensatzes: 13.03.2019.

LFG - Luftfahrtgesetz (1957): Bundesgesetz über die Luftfahrt. Fassung vom 22.03.2019. RIS - Rechtsinformationssystem des Bundes. Österreich.

Lula, K., Duane Nellis, M. und Rundquist, B. (2012): Celebrating 40 years of Landsat program's Earth observation accomplishments. Geocarto International (27:6): 459-459.

Maggiori E., Tarabalka Y., Charpiat G. und Alliez P. (2017): Can semantic labeling methods generalize to any city? the inria aerial image labeling benchmark. In: 2017 IEEE International 
Geoscience and Remote Sensing Symposium (IGARSS), IEEE, pp. 3226-9.

Mair, M., Zischg, J., Rauch, W. und Sitzenfrei, R. (2017): Where to Find Water Pipes and Sewers?-On the Correlation of Infrastructure Networks in the Urban Environment. Water 9(2), 146 .

Marmanis, D., Schindler, K., Wegner, J. D., Galliani, S., Datcu, M. und Stilla, U. (2018): Classification with an edge: Improving semantic image segmentation with boundary detection. ISPRS Journal of Photogrammetry and Remote Sensing 135, 158-72.

Matzinger, A., Riechel, M., Remy, C., Schwarzmïller, H., Rouault, P., Schmidt, M., Offermann, M., Strehl, C., Nickel, D., Sieker, H. Pallasch, M., Köhler, M., Kaiser, D., Möller, C. Büter, B., Leßmann, D., von Tils, R., Säumel, I. Pille, L., Winkler, A., Bartel, H., Heise, S., Heinzmann, B., Joswig, K., Rehfeld-Klein, M. und Reichmann, B. (2017): Zielorientierte Planung von Maßnahmen der Regenwasserbewirtschaftung - Ergebnisse des Projektes KURAS. Berlin.

MicroImages. (2015): Getting Started with TNTmips, TNTedit and TNTview. Webzugriff: https:// www.microimages.com/documentation/Tutorials GetStartTNT.pdf [Zuletzt Besucht am: 09.04.2019] Moy de Vitry, M., Schindler, K., Rieckermann, J. Leitão, J. P. (2018): Sewer inlet localization in uav image clouds: Improving performance with multiview detection. Remote Sensing 10, 706. Oberascher, M., Zischg, J., Palermo, S. A., Kinzel, C., Rauch, W. und Sitzenfrei, R. (2019): Smart Rain Barrels: Advanced LID Management Through Measurement and Control. In: New Trends in Urban Drainage Modelling. UDM 2018. Green Energy and Technology. G. M (ed.), Springer, Cham.

Pajares, G. (2015): Overview and current status of remote sensing applications based on unmanned aerial vehicles (UAVs). Photogrammetric Engineering \& Remote Sensing Vol. 81, No. 4: 281-329.

Puente, I., González-Jorge, H., Martínez-Sánchez, J., und Arias, P. (2013): Review of mobile mapping and surveying technologies. Measurement, 46(7): 2127-2145.
QGIS Development Team (2019): QGIS Geographic Information System. Open Source Geospatial Foundation Project. Web-Zugriff auf: http:// qgis.osgeo.org [Zuletzt Besucht am: 08.04.2019]. Rauch, W., Urich, C., Bach, P.M., Rogers B.C. de Haan, F.., Brown, R.R., Mair, M., McCarthy, D.T., Kleidorfer, M., Sitzenfrei, R. und Deletic, A. (2017): Modelling transitions in urban water systems. Water Research 126, Elsevier, 501-514. Rossman, L. A. (2015): Storm water management model user's manual, version 5.1. Nationa Risk Management Research Laboratory, Office of Research and Development, US Environmental Protection Agency.

Satellogic (2019): About Satellogic. Web-Zugriff auf: https://satellogic.com/government/ [Zuletzt Besucht am: 12.03.2019].

See, L., Mooney, P., Foody, G., Bastin, L., Comber, A., Estima, J., Fritz, S., Kerle, N., Jiang, B. Laakso, M., Liu, H.-Y., Milčinski, G., Nikšič, M. Painho, M., Pődör, A., Olteanu-Raimond, A. M. und Rutzinger, M. (2016): Crowdsourcing, Citizen Science or Volunteered Geographic Information? The Current State of Crowdsourced Geographic Information. ISPRS International Journal of Geo-Information. Vol. 5(5): 1-23. Simperler, L., Himmelbauer, P., Stöglehner, G. and Ertl, T. (2018): Siedlungswasserwirtschaftliche Strukturtypen und ihre Potenziale für die dezentrale Bewirtschaftung von Niederschlagswasser. Österreichische Wasser- und Abfallwirtschaft, Wien.

Sitzenfrei, R., Kleidorfer, M., Meister, M., Burger, G., Urich, C., Mair, M., und Rauch, W. (2014): Scientific computing in urban water management. In Computational engineering (pp. 173-193). Springer, Cham.

Magistrat der Stadt Wien, Wiener Umweltschutzabteilung (2010): Integratives Regenwassermanagement: Beispielsammlung. [Writing Team, Grimm, K. and Achleitner, M.]. Wien. Stadt Wien und Österreichische Länder bzw. Ämter der Landesregierung (2016): Basemap.at - Verwaltungsgrundkarte von Österreich. WebZugriff auf: https://basemap.at/ [Zuletzt Besucht am: 18.03.2019]
Stadtentwässerungsbetriebe (StEB) Köln (2017) Leitfaden für eine wassersensible Stadt-und Freiraumgestaltung in Köln. Köln.

Toth, C. und Józków, G. (2016): Remote sensing platforms and sensors: A survey. ISPRS Journal of Photogrammetry and Remote Sensing (115): 22-36.

Tscheikner-Gratl, F., Mikovits, C., Sitzenfrei, R., Rauch, W. und Kleidorfer, M. (2015): GIS-Anwendung in der integrierten Rehabilitierungsplanung von urbaner Wasserinfrastruktur. In: Strobl, J., Zagel, B., Griesebner, G., Blaschke, T. Angewandte Geoinformatik 2015: Beiträge zum 27. AGIT-Symposium Salzburg. Heidelberg: Wichmann, ISBN 978-3-87907-557-7, S. 308-313. UNOOSA-United Nations Office for Outer Space Affairs (2019): Online Index of Objects Launched into Outer Space. Web-Zugriff auf: http://www.unoosa.org/oosa/osoindex/searchng.jspx?lf_id= [Zuletzt Besucht am: 12.03.2019]. Wallace, L., Lucieer, A., Watson, C., und Turner, D. (2012): Development of a UAV-LiDAR system with application to forest inventory. Remote Sensing, 4(6): 1519-1543.

Warsta, L., Niemi, T. J., Taka, M., Krebs, G. Haahti, K., Koivusalo, H. und Kokkonen, T (2017): Development and application of an automated subcatchment generator for SWMM using open data. Urban Water Journal 14(9), 954-63.

Woods Ballard, B., Dimova, G., Weisgerber, A., Kellagher, R., Abbot, C., Maneiro Franco, E., Smith, H. und Stovin, V. (2005): Benefits and Performance of Sustainable Drainage Systems. Project Report. HR Wallingford.

Zischg, J., Zeisl, P., Winkler, D., Rauch, W. und Sitzenfrei, R. (2018): On the sensitivity of geospatial Low Impact Development location to the centralized sewer network. Water science and technology 77(7), 10.

Hinweis des Verlags Der Verlag bleibt in Hinblick auf geografische Zuordnungen und Gebietsbezeichnungen in veröffentlichten Karten und Institutsadressen neutral. 